\title{
The proposed scoring system for hospitalization or discharge of patients with COVID-19
}

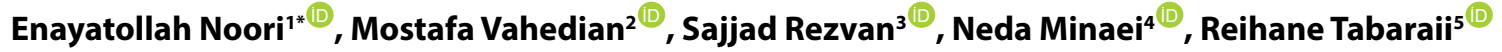 \\ 'General Practitioner, Qom University of Medical Sciences, Qom, Iran \\ 2Department of Family and Community Medicine, School of Medicine, Qom University of Medical Sciences, Qom, Iran \\ ${ }^{3}$ Rafsanjan University of Medical Sciences, Rafsanjan, Iran \\ ${ }^{4}$ Radiology Department of Medical Research Committee, Qom University of Medical Sciences, Qom, Iran \\ ${ }^{5}$ Department of Internal Medicine, School of Medicine, Shahid Beheshti Hospital, Qom University of Medical Sciences, Qom, Iran
}

\author{
Received: 4 October 2020 \\ Accepted: 18 January 2021 \\ Published online: 30 January 2021 \\ *Corresponding author: \\ Enayatollah Noori, Institutional \\ details: General Practitioner, Qom \\ University of Medical Sciences, Qom, \\ Iran, Iran, \\ Tel: + 982536122949 , \\ Fax:+982536122945, \\ Email: Enoori@muq.ac.ir \\ Competing interests: None. \\ Funding information: None \\ Citation: Noori E, Vahedian M, \\ Rezvan S, Minaei N, Tabaraii R. \\ The proposed scoring system for \\ hospitalization or discharge of \\ patients with covid-19. Journal of \\ Emergency Practice and Trauma \\ 2022; 8(1): 60-63. doi: 10.34172/ \\ jept.2021.08
}

\begin{abstract}
Objective: Since the outbreak of coronavirus disease 2019 (COVID-19), the triage of patients diagnosed with corona virus has been a very important issue. The aim of this study was to introduce a triage scoring system according to the clinical and para-clinical findings of patients in order to be admitted or discharged with COVID-19.

Methods: After confirming the positive polymerase chain reaction (PCR) test for patients, we used a scoring system which included: the age of patient (less than 40 years and $>40$ years), early vital signs at the time of admission, lab tests including C-reactive protein (CRP), white blood count (WBC), lactate dehydrogenase (LDH), D-dimer, chest imaging findings, comorbidity and shortness of breath.

Results: The clinical score obtained for each variable in this scoring system was a number between 0 and 3. The total score was a minimum of 0 and a maximum of 17. A higher score indicated an increase in the intensity and the need for intensive care. These scores were classified into 3 groups: 0-4, 5-10 and above 10. In the next stage, patients were divided into three groups: mild, moderate and severe. In this regard, patients with mild symptoms were suggested to receive home quarantine and home treatment, patients with moderate symptoms were recommended hospitalization and medical care, and finally patients with severe symptoms were inclined to intensive care.

Conclusion: In order to treat and manage patients with COVID-19, it is necessary to pay particular attention to clinical and para-clinical findings and prioritize these findings based on the severity and the condition of patients.

Keywords: COVID-19, Scoring system, hospitalization, discharge, Triage
\end{abstract}

\section{Introduction}

Coronavirus 2019, caused by a common humananimal virus called severe acute respiratory syndrome coronavirus 2 (SARS-CoV-2), has been recently emerged (1). The disease has spread as a pandemic in all countries of the world (2). It is very important to know the symptoms of this disease. However, the clinical symptoms may be nonspecific $(3,4)$. Elderly people and those with underlying diseases develop acute respiratory symptoms due to alveolar destruction (5). This disease can advance to the progressive involvement of several organs of the body and can even quickly cause the death of patients (6). Changes in laboratory signs include white blood cell count, lymphopenia, and thrombocytopenia as well as increased prothrombin time $(\mathrm{PT})$ and C-reactive protein (CRP) $(3,5)$. Patients with mild clinical symptoms may not need to be hospitalized at first. However, clinical signs and symptoms may take several days after the onset of the disease to progress to the lower parts of the lungs to be worsened. In this situation, all patients should be closely monitored. Risk factors known for the patients to develop the most ferocious conditions include old age, underlying diseases, weak immune system and pregnant women (7). The decision to monitor and care for the patient in an inpatient or outpatient setting should be made on a caseby-case basis. In addition to clinical manifestations, the decision depends on the patients' ability to quarantine and take care of themselves, as well as the possibility of involving other family members in the disease (8). After visiting the doctor and performing the necessary diagnostic and therapeutic measures, as well as medical consultations in other specialized fields (if necessary), patients are classified in several categories. Uncomplicated patients will be discharged from the emergency department 
with the necessary training and follow-up consultations. Specific forms for these patients will be completed and their contact information (telephone number and address) will be registered. For patients with a diagnosis of pneumonia, hospitalization in the relevant ward with physical isolation conditions using caution/drop caution is taken into account. Patients with severe pneumonia who are candidates for admission to special wards will be admitted to the hospital after coordination. In the absence of a special bed and after the necessary coordination with the responsible physician, the patient will be sent to a ready hospital with empty beds. In short, due to the crisis and the quick transmission of the disease as well as the need for facilities such as special beds and isolation in hospitals, paying particular attention to the clinical course and manifestations of the disease in patients is necessary. In this regard, providing efficient care and management of patients seems critical. To accomplish this goal, having accurate criteria for hospitalization and management of patients with COVID-19 is essential. To the best of our knowledge, there is currently no exact standard for hospitalization of these patients. Therefore, the aim of this study was to introduce a triage scoring system according to the clinical and para-clinical findings of patients in order to be admitted or discharged with COVID-19.

\section{Methods}

This system of scoring was used to evaluate patients diagnosed with COVID-19 admitted in the hospital. After confirming the positive polymerase chain reaction (PCR) test for patients, based on the patients' clinical and paraclinical findings, and by using the table below, we gave patients a specific score. In this regard, we could decide whether to admit or discharge the patients based on the severity of the disease (Table 1). In this scoring system, the age of the patient (less than 40 years and $>40$ years), early vital signs at admission, lab tests including WBC, differential count, CRP, lactate dehydrogenase (LDH), D-dimer, chest imaging findings, underlying chronic disease and shortness of breath were assessed (Table 2).

\section{Results}

The clinical score obtained for each variable in this scoring system was a number between 0 and 3 . The total score was a minimum of 0 and a maximum of 17 . A higher score indicates an increase in the intensity and the need for intensive care. This score was classified into 3 groups: $0-5,6-11$ and above 11 (Table 2). In the next stage, patients were divided into green (discharge and recovery), yellow (remain in hospital) and red (died) groups. The recommendation for patients in the mild group was discharge, treatment at home and quarantine). Also, in the moderate group, hospitalization and treatment were suggested. In the severe group, ICU admission was recommended (Table 1). Finally, a pyramid for hospitalization or discharge of patients was taken into account.

\section{Discussion}

In this study, a scoring system was designed and according to this system, during the pandemic of COVID-19, patients can be triaged based on the clinical conditions as

Table 1. Scoring system and color coding triage system

\begin{tabular}{lc}
\hline Parameter & Outcome \\
\hline $\begin{array}{l}\text { Quarantine and care at home until symptoms } \\
\text { improve }\end{array}$ & Discharge and recovery \\
\hline Admitted to ward & Remained in hospital \\
ICU admission and caring in critical care unit & Died \\
\hline
\end{tabular}

Table 2. Suggested scoring for hospitalization or discharge of patients with COVID-19

\begin{tabular}{|c|c|c|}
\hline Parameter & & Score \\
\hline \multirow{2}{*}{ Age } & Under 40 & 0 \\
\hline & Over 40 & 1 \\
\hline \multirow{2}{*}{ Comorbidities } & No & 0 \\
\hline & Yes & 1 \\
\hline \multirow{5}{*}{ Respiratory rate } & $\leq 8$ & 3 \\
\hline & $9-11$ & 1 \\
\hline & $12-20$ & 0 \\
\hline & $21-24$ & 2 \\
\hline & $\geq 25$ & 3 \\
\hline \multirow{5}{*}{ Heart rate, } & $60-100$ & 0 \\
\hline & $40-60$ & 1 \\
\hline & Under 40 & 2 \\
\hline & $100-130$ & 1 \\
\hline & Over 130 & 2 \\
\hline \multirow{4}{*}{ Spo2 } & $\leq 91 \%$ & 3 \\
\hline & $92-93 \%$ & 2 \\
\hline & $94-95 \%$ & 1 \\
\hline & $\geq 96 \%$ & 0 \\
\hline \multirow{3}{*}{ Temperature } & $36.1-38$ & 0 \\
\hline & 38.1-39 & 1 \\
\hline & $\geq 39.1$ & 2 \\
\hline \multirow{2}{*}{ CXR or CT abnormal } & no & 0 \\
\hline & yes & 1 \\
\hline \multirow{5}{*}{ SBP } & $\leq 90$ & 3 \\
\hline & $91-100$ & 2 \\
\hline & $101-110$ & 1 \\
\hline & $111-219$ & 0 \\
\hline & $\geq 220$ & 3 \\
\hline \multirow[b]{2}{*}{ Lab date } & Normal & 0 \\
\hline & $\begin{array}{l}\text { Leucopenia, Lymphopenia } \leq 1500 \text {, } \\
\text { CRP }>10 \text {, LDH }>250 \text {, D-dimer }>0.5\end{array}$ & 1 \\
\hline
\end{tabular}


well as hospital facilities and capacity including hospital beds and equipment such as ventilators.

In this system, the infiltration findings and ground glass lesions of chest CT scan and radiography are diagnostic for the COVID-19. Furthermore, we took the patients' medical history at the admission time, such as respiratory rate, respiratory distress, and SPo2. The crucial labfindings are lymphopenia, leukopenia, LDH, CRP, high D-dimer, which have the particular value due to the inflammatory conditions in COVID 19.

The findings of these studies are compared with the results of the present study. The difference between our study and the mentioned studies is the scoring system for the admission of patients. In our system, all clinical and para-clinical findings are taken into consideration and patients are categorized into three groups: low-risk group (green), moderate-risk group (yellow), and highrisk group (red). Depending on the condition of these patients, home care program, care and hospitalization in the general ward or hospitalization in intensive care as well as support for these patients can be considered. By using this scoring system, the clinical condition of each patient is recognized and physicians can better understand the patient's condition and track the respiratory complication rate. In addition, doctors can also see patients at a specific point (for example, the second level which may require intubation). In general, it can be said that by the use of this type of triage system, and assigning color codes to patients according to their clinical conditions, treatment priorities can be used more logically. One of the advantages of this scoring system is the prioritization of patients in critical conditions to transfer them to the intensive care unit in a timely manner. In developing countries, medical resources including the number of ICU beds and ventilators are among the limitations of treatment and can be used in global epidemics.

Research has been done in the field of triage of COVID-19 patients. The study conducted by Salunke et al revealed the use of variables such as age, body temperature, cough and underlying diseases in the triage system (9). In another study, apart from the mentioned variables, patients' blood pressure was also considered (10). In the study undertaken by Duca et al. (11), important respiratory symptoms such as wheezing, $\mathrm{SPo}$, and chest radiographic findings were used to triage COVID-19 patients. In two other studies, age, laboratory findings and underlying diseases had a role in the condition of patients and their hospitalization $(13,14)$. The limitation of this system is its invalidity. However, in future studies, this system may be validated and, if approved, it can be used effectively in medical resources.

\section{Conclusion}

This system is useful in better management of patients with COVID-19. This system includes clinical and paraclinical findings. We suggest a multicenter study to validate all existing scoring systems.

\section{Authors' contributions}

Each author contributed equally to the study design, drafting the article, reading critically and accepted the final proof.

\section{Ethical issues}

This study was approved by Qom University of Medical Sciences (ID: IR.MUQ.REC.1398.153).

\section{References}

1. Zhu N, Zhang D, Wang W, Li X, Yang B, Song J, et al. A novel coronavirus from patients with pneumonia in China, 2019. N Engl J Med. 2020; 382(8): 727-33. doi: 10.1056/ NEJMoa2001017.

2. Salunke AA, Nandy K, Pathak SK, Shah J, Kamani M, Kotakotta V, et al. Impact of COVID-19 in cancer patients on severity of disease and fatal outcomes: a systematic review and meta-analysis. Diabetes Metab Syndr. 2020; 14(5): 1431-7. doi: 10.1016/j.dsx.2020.07.037.

3. Guan WJ, Ni ZY, Hu Y, Liang WH, Ou CQ, He JX, et al. Clinical characteristics of 2019 novel coronavirus infection in China. MedRxiv. 2020. doi: 10.1101/2020.02.06.20020974.

4. Wang D, Hu B, Hu C, Zhu F, Liu X, Zhang J, et al. Clinical characteristics of 138 hospitalized patients with 2019 novel coronavirus-infected pneumonia in Wuhan, China. JAMA. 2020; 323(11): 1061-1069. doi: 10.1001/jama.2020.1585.

5. Chen N, Zhou M, Dong X, Qu J, Gong F, Han Y, et al. Epidemiological and clinical characteristics of 99 cases of 2019 novel coronavirus pneumonia in Wuhan, China: a descriptive study. Lancet. 2020; 395(10223): 507-13. doi: 10.1016/S0140-6736(20)30211-7.

6. Huang C, Wang Y, Li X, Ren L, Zhao J, Hu Y, et al. Clinical features of patients infected with 2019 novel coronavirus in Wuhan, China. Lancet. 2020; 395(10223): 497-506. doi: 10.1016/S0140-6736(20)30183-5.

7. CDC. Interim infection prevention and control recommendations for hospitalized patients with Middle East respiratory syndrome coronavirus (MERS-CoV). Availeble from: https://www.cdc.gov/coronavirus/mers/ infection-prevention-control.html. [cited 2015 Jul 20]. 2017.

8. Memberships M, Join T. Guidance on Coronavirus Disease 2019 (COVID-19) for Transplant Clinicians. Availeble from: https://tts.org/23-tid/tid-news/657-tid-update-andguidance-on-2019-novel-coronavirus-2019-ncov-fortransplant-id-clinicians. Updated 8 June 2020.

9. Salunke AA, Pathak SK, Dhanwate A, Warikoo V, Nandy $\mathrm{K}$, Mendhe $\mathrm{H}$, et al. A proposed $\mathrm{ABCD}$ scoring system for patient's self assessment and at emergency department with symptoms of COVID-19. Diabetes Metab Syndr. 2020; 14(5): 1495-501. doi: 10.1016/j.dsx.2020.07.053.

10. Shi Y, Yu X, Zhao H, Wang H, Zhao R, Sheng J. Host susceptibility to severe COVID-19 and establishment of a host risk score: findings of 487 cases outside Wuhan. Crit Care. 2020;24(1):108. doi: 10.1186/s13054-020-2833-7. 
11. Duca A, Piva S, Focà E, Latronico N, Rizzi M. Calculated decisions: Brescia-COVID Respiratory Severity Scale (BCRSS)/algorithm. Emerg Med Pract. 2020; 22(5 Suppl): CD1-2.

12. Ji D, Zhang D, Xu J, Chen Z, Yang T, Zhao P, et al. Prediction for progression risk in patients with COVID-19 pneumonia: the CALL Score. Clin Infect Dis. 2020; 71(6): 1393-9. doi: 10.1093/cid/ciaa414.
13. Wallis LA. COVID-19 Severity Scoring Tool for low resourced settings. Afr J Emerg Med. 2020. doi: 10.1016/j. afjem.2020.03.002.

14. Hu H, Yao N, Qiu Y. Comparing rapid scoring systems in mortality prediction of Critically Ill patients with novel coronavirus disease. Acad Emerg Med. 2020; 27(6): 461-8. doi: 10.1111/acem.13992. 